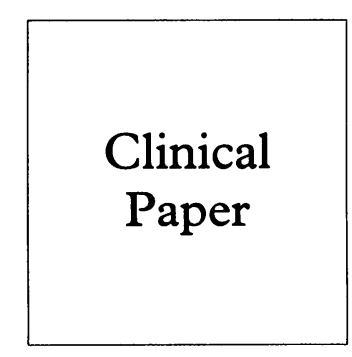

\title{
The chronically symptomatic vulva: prevalence in primary health care
}

\author{
David Nunns, Deb Mandal
}

Objectives: To examine within the primary health care setting, the frequency of chronic vulval disorders, their frequency, investigation, management and referral patterns.

Methods: A questionnaire based survey at general practitioners surrounding a district general hospital.

Results: A total of 79 (72\%) GPs replied to the questionnaire. Thirty-six (45\%) GPs saw more than one patient per month with recurrent vulval symptoms and ten (13\%) GPs saw more than three patients per month. The predominant symptom amongst patients with recurrent vulval symptoms was itching $(55 \%)$ followed by soreness $(26 \%)$, burning (14\%) and dyspareunia (5\%). Topical-antifungals and steroid medications were the commonest treatments prescribed for all women with vulval symptoms.

Conclusions: Women who have vulval complaints commonly attend their GP for treatment. In many instances women with chronic vulval complaints are inadequately investigated and inappropriately managed.

(Genitourin Med 1996;72:343-344)

Keywords: vulva; management; general practice

\section{Introduction}

The management of women with chronic benign vulval conditions is one of the most difficult and challenging aspects of women's health care today. In 1985 the International Society for the Study of Vulvar Diseases attempted to provide a comprehensive classification of these conditions and the term vulvodynia was ascribed to women with chronic vulval discomfort, characterised by burning, stinging, rawness or irritation in the absence of overt infection or dermatoses. ${ }^{1}$ In this paper, we present our results of a postal survey directed at general practitioners (GPs) in an attempt to assess the scale of the problem in the primary health care setting.

\section{Methods}

A postal questionnaire was sent to 110 local GPs in the area surrounding Bolton General Hospital. Bolton General hospital is a district general hospital set in the North-West of England and serves a stable population of approximately 250000 people. Questions were asked about the numbers of women presenting with vulval conditions, the first line of investigation and treatment offered to patients with recurrent vulval problems and which specialities patients were referred to. The questionnaire did not address the way GPs counselled their patients when presenting with symptoms. A recurrent vulval disorder was defined as patients presenting with vulval symptoms on different clinical episodes or ongoing symptoms of varying degree without response to treatment. This clinical description of vulval complaints was made in preference to the International Society of Vulvar Diseases classification of which many GPs are not aware. ${ }^{2}$

\section{Results}

A total of 79 (72\%) GPs replied to the questionnaire. The frequency of vulval complaints presenting to GPs was high with $41(52 \%)$ seeing more than three patients per month. Patients with recurrent symptoms were seen less often with $36(46 \%)$ GPs seeing one patient per month, however, ten (13\%) GPs saw more than three patients per month. The table summarise the numbers of patients attending GPs with any vulval symptoms and recurrent vulval symptoms. The predominant symptom amongst patients with recurrent vulval symptoms was itching $(55 \%)$ followed by soreness $(26 \%)$, burning (14\%) and dyspareunia $(5 \%)$. Genital examinations were always carried out on patients with recurrent symptoms by $44(56 \%)$ GPs. Thirty-five GPs occasionally or never examined patients with recurrent symptoms. The reasons given by GPs for not examining patients were if the history suggested the diagnosis, a lack of time in the surgery and at the request of the patient.

Only $59 \%$ of patients with recurrent symptoms were investigated prior to treatment. Of the rest, twenty-three (29\%) GPs occasionally investigated patients and nine (11\%) did not investigate patients at all. A high vaginal swab was the commonest investigation carried out by 66 GPs, 42 performed chlamydia swabs and 9 performed urinalysis. Only one GP performed the amine test.

The remainder of the questionnaire concerned those patients with recurrent vulval disorders where no diagnosis was made microbiologically or dermatologically. In this group, patients with itching as the predominant symptom were commonly diagnosed empirically as having candidiasis $(n=54$, $68 \%$ ) and were all treated with topical antifungals. In 29 cases this treatment was supple- 
Number of patients seen per month with vulval symptoms

\begin{tabular}{lll}
\hline Number of patients seen per month & $\begin{array}{l}\text { Number of GPs (\%) } \\
\text { General vulval } \\
\text { symptoms }\end{array}$ & $\begin{array}{l}\text { Recurrent vulval } \\
\text { symptoms * }\end{array}$ \\
\hline$>3$ & $41(52)$ & $10(13 \%)$ \\
3 & $14(18)$ & $5(6 \%)$ \\
2 & $15(19)$ & $16(20 \%)$ \\
1 & $8(10 \%)$ & $36(46 \%)$ \\
0 & $1(1 \%)$ & $12(15 \%)$ \\
\hline
\end{tabular}

*A recurrent vulval disorder was defined as patients presenting with vulval symptoms on different clinical episodes or ongoing symptoms of varying degree without response to treatment.

mented with steroid creams and in 11 cases with metronidazole. Vulvar vestibulitis, a painful inflammatory disorder of the vestibule, was the second commonest diagnosis in this group, made in 7 cases $(9 \%)$. No diagnosis was given by four GPs. Similarly, the commonest diagnosis amongst those patients who predominantly complained of burning, was again candidiasis $(n=25)$. These patients were all treated with topical antifungals, sometimes in combination with topical steroids $(n=3)$ and metronidazole $(n=3)$. Vulvar vestibulitis was the second commonest diagnosis made in this group made in 8 cases. Twenty-one GPs could not make any diagnosis with burning as the predominant symptom and all these patients were referred to various specialists. Finally, there was a diverse range of clinical diagnoses made amongst those patients with superficial dyspareunia as the predominant symptom. Candidiasis was the commonest diagnosis given to patients in 14 cases and in 12 cases patients were labelled as having "pressure symptoms". Twenty-eight GPs had no diagnosis for these patients and in 15 cases no treatment was prescribed although all GPs referred patients to specialists.

All but one GP referred patients to various specialists. Fifty-nine GPs $(75 \cdot 6 \%)$ referred patients to more than one specialist. This was commonly a genitourinary medicine physician, gynaecologist or dermatologist. Only 20 GPs referred to one specialist and this was a gynaecologist in 17 cases and a genitourinary medicine physician in 3 cases.

\section{Discussion}

Women with vulval complaints commonly attend their GP for treatment. The frequency of vulval conditions has not been assessed in this setting in the past and our results indicate that the workload on some GPs may be con- siderable in dealing with this problem. In this survey, over half the GPs who responded saw more than three patients a month in their practice. This work-load is further increased as each patient requires a long consultation period for a history, examination and counselling. In many cases $(n=35)$, patients with recurrent symptoms were occasionally or never examined with a lack of time as being a contributory factor.

It is of concern that many patients were not examined or investigated when presenting with symptoms. A detailed vulval examination will identify clinically obvious vulval dermatoses and vulvar vestibulitis. Recurrent candidiasis also should only be treated if confirmed microbiologically. Several distinct subsets of vulvodynia have been identified. ${ }^{3}$ These include (1) vulvar vestibulitis, (2) vulvar dermatoses, (3) psychosomatic vulvovaginitis, (4) recurrent candidiasis and (5) dysaesthetic vulvodynia. These subsets may occur alone, simultaneously or sequentially. Of these sub-groups, vulvar vestibulitis is the commonest condition and the most difficult to treat. No diagnosis was given by GPs to most patients with superficial dyspareunia as the predominant symptom; however, this can be the predominant symptom in women with vulvodynia especially vulvar vestibulitis, one of the most common subgroups of vulvodynia.

Benign, recurrent vulval disorders have long been neglected in medicine, yet conditions like vulvodynia have been documented for more than a century. ${ }^{4}$ Only recently have specialised vulval clinics been set-up to deal with the problem; however, there are only a handful currently in operation in the UK. This questionnaire has highlighted the scale of recurrent vulval disorders in the primary health care setting and the considerable demands on the GP with these patients. There are no easy options for managing recurrent vulval conditions and education, awareness and research is urgently needed into this troublesome condition.

We acknowledge the help of Upjohn Pharmaceuticals in distributing this questionnaire.

1 Lynch PJ. Vulvodynia: A syndrome of unexplained vulvar pain, psychological disability and sexual dysfunction. $f$ Reprod Med 1985;31:773-80.

2 Ridley CM. ISSVD New Nomenclature for Vulval Disease. Am $\mathcal{F}$ Obstet Gynecol 1989;100:769-70.

3 McKay M. Subsets of vulvodynia. $\mathcal{F}$ Reprod Med 1988;33: 695-7.

4 Skene AJC. Treatise on the Diseases of Women. New York: Appelton and Company 1889. 